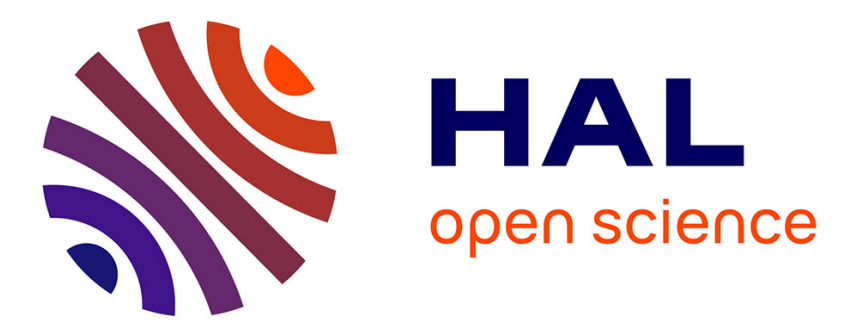

\title{
Energy boost in laser wakefield accelerators using sharp density transitions
}

A. Döpp, E. Guillaume, C. Thaury, Agustin Lifschitz, Kim Ta Phuoc, Victor

Malka

\section{- To cite this version:}

A. Döpp, E. Guillaume, C. Thaury, Agustin Lifschitz, Kim Ta Phuoc, et al.. Energy boost in laser wakefield accelerators using sharp density transitions. Physics of Plasmas, 2016, 23 (5), pp.56702. 10.1063/1.4946018 . hal-01342480

\section{HAL Id: hal-01342480 \\ https://hal.science/hal-01342480}

Submitted on 11 Oct 2017

HAL is a multi-disciplinary open access archive for the deposit and dissemination of scientific research documents, whether they are published or not. The documents may come from teaching and research institutions in France or abroad, or from public or private research centers.
L'archive ouverte pluridisciplinaire HAL, est destinée au dépôt et à la diffusion de documents scientifiques de niveau recherche, publiés ou non, émanant des établissements d'enseignement et de recherche français ou étrangers, des laboratoires publics ou privés. 


\section{Energy boost in laser wakefield accelerators using sharp density transitions}

A. Döpp, E. Guillaume, C. Thaury, A. Lifschitz, K. Ta Phuoc, and V. Malka

Citation: Physics of Plasmas 23, 056702 (2016); doi: 10.1063/1.4946018

View online: http://dx.doi.org/10.1063/1.4946018

View Table of Contents: http://aip.scitation.org/toc/php/23/5

Published by the American Institute of Physics

\section{Articles you may be interested in}

Giga-electronvolt electrons due to a transition from laser wakefield acceleration to plasma wakefield acceleration

Physics of Plasmas 21, 123113 (2014); 10.1063/1.4903851

External injection and acceleration of electron bunch in front of the plasma wakefield produced by a periodic chirped laser pulse

Physics of Plasmas 24, 013110 (2017); 10.1063/1.4973662

Generation of $20 \mathrm{kA}$ electron beam from a laser wakefield accelerator

Physics of Plasmas 24, 023108 (2017); 10.1063/1.4975613

Injection and acceleration of quasimonoenergetic relativistic electron beams using density gradients at the edges of a plasma channel

Physics of Plasmas 17, 083107 (2010); 10.1063/1.3469581

Staging of laser-plasma accelerators

Physics of Plasmas 23, 056705 (2016); 10.1063/1.4948280

3D printing of gas jet nozzles for laser-plasma accelerators

Review of Scientific Instruments 87, 073505 (2016); 10.1063/1.4958649

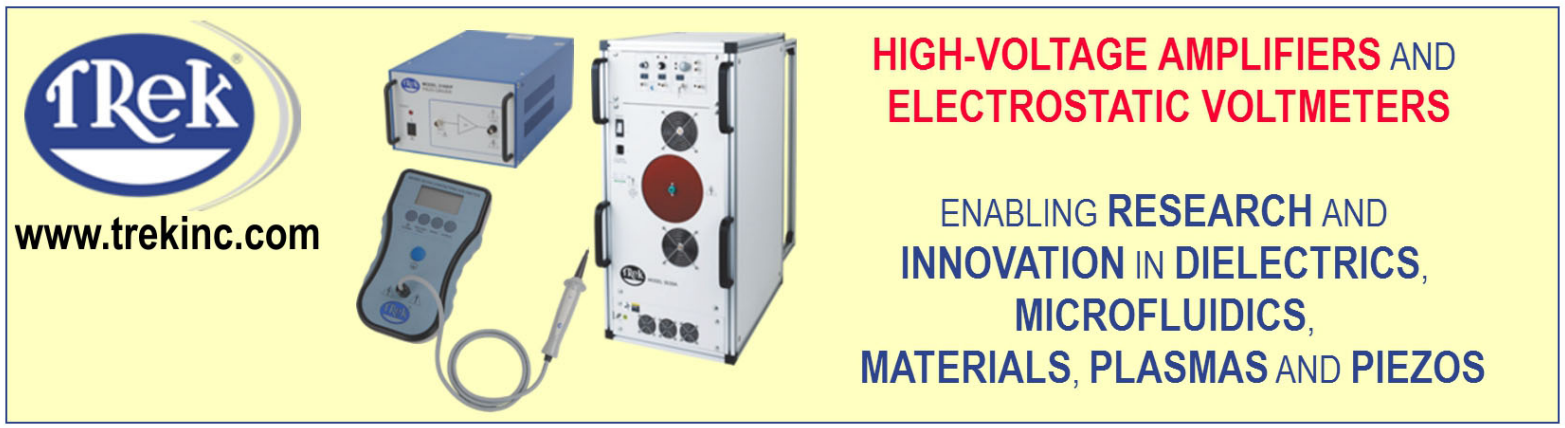




\title{
Energy boost in laser wakefield accelerators using sharp density transitions
}

\author{
A. Döpp, E. Guillaume, C. Thaury, ${ }^{\text {a) }}$ A. Lifschitz, K. Ta Phuoc, and V. Malka \\ LOA, ENSTA ParisTech—CNRS-École Polytechnique-Université Paris-Saclay, \\ 828 Boulevard des Maréchaux, 91762 Palaiseau Cedex, France
}

(Received 18 December 2015; accepted 24 February 2016; published online 13 April 2016)

\begin{abstract}
The energy gain in laser wakefield accelerators is limited by dephasing between the driving laser pulse and the highly relativistic electrons in its wake. Since this phase depends on both the driver and the cavity length, the effects of dephasing can be mitigated with appropriate tailoring of the plasma density along propagation. Preceding studies have discussed the prospects of continuous phaselocking in the linear wakefield regime. However, most experiments are performed in the highly nonlinear regime and rely on self-guiding of the laser pulse. Due to the complexity of the driver evolution in this regime, it is much more difficult to achieve phase locking. As an alternative, we study the scenario of rapid rephasing in sharp density transitions, as was recently demonstrated experimentally. Starting from a phenomenological model, we deduce expressions for the electron energy gain in such density profiles. The results are in accordance with particle-in-cell simulations, and we present gain estimations for single and multiple stages of rephasing. Published by AIP Publishing.

[http://dx.doi.org/10.1063/1.4946018]
\end{abstract}

\section{INTRODUCTION}

Plasma-based particle accelerators ${ }^{1}$ use charge separation between electrons and ions to create electric fields in the order of gigavolt to teravolt per meter, exceeding the breakdown-limited field strength in conventional accelerators by several orders of magnitude. The charge separation is induced by a driver, which is either a bright particle or laser beam. The former is known as beam-driven plasma wakefield acceleration (PWFA), which could notably demonstrate energy doubling of a $42 \mathrm{GeV}$-class electrons beam ${ }^{2}$ as well as positron acceleration. ${ }^{3}$ The latter is referred to as laser wakefield acceleration (LWFA), and during the past two decades, this method has been used to accelerate electrons from rest to first some tens of $\mathrm{MeV},{ }^{4}$ to always higher energies, ${ }^{5}$ reaching $\mathrm{GeV}$-scale, ${ }^{6}$ and recently multi-GeV energies. ${ }^{7-9}$

Plasma wakefield acceleration can be seen as a special type of resonance acceleration, whose accelerating structure is a plasma wave. In this kind of accelerator, a particle first goes through an injection process, whose primary challenge is to make the particles co-propagate with the wave. While PWFA experiments usually inject part of the drive beam into the wakefield, LWFA almost exclusively relies on the injection of background plasma electrons. Such injection can be facilitated using, for instance, density downramps, ${ }^{10,11}$ colliding pulses, ${ }^{12}$ or delayed tunneling ionization. ${ }^{13}$

Once injected the interaction length is essentially limited to the length over which the wave structure can be sustained. While this condition assures that energy exchange between the wave and particles is possible, it is also important to assure that particles interact with the accelerating part of the field, ideally maintaining a synchronous phase $\phi$ with the strongest possible field gradients. Initially, this is the

Note: Paper TI3 5, Bull. Am. Phys. Soc. 60, 306 (2015).

${ }^{a)}$ Invited speaker. case in most injection scenarios, as particles are usually trapped at the very back of the wakefield. However, for such phase matching, the particle velocity $v_{e}$ and the phase velocity of the wave $v_{\phi}$ need to be the same, which is not necessarily the case.

In beam-driven plasma wakefield accelerators, both driver and witness are highly relativistic, i.e., the associated Lorentz factor $\gamma \gg 1$. Both are therefore moving at a velocity close to the speed of light in vacuum $c_{0}$ (approximately $\left.v_{e} / c_{0} \approx 1-1 / 2 \gamma^{2}\right)$, and the dephasing is not a pressing issue. In travelling wave RF accelerators, the phase velocity is, in general, superluminal, and the synchronization problem is often resolved by disk-loading the cavities, which reduces the phase velocity. ${ }^{14}$ However, in laser-driven wakefield acceleration, dephasing remains the mayor limitation of achievable energy gain. Here, the phase velocity of the plasma wave is of the order of the group velocity of the laser driver, which for a cold underdense plasma is

$$
\frac{v_{g}}{c_{0}} \approx 1-\frac{1}{2} \frac{n_{e}}{n_{c}}
$$

where $n_{e} / n_{c} \ll 1$ denotes the ratio of electron density $n_{e}$ with respect to the critical density at a laser wavelength $\lambda_{0}\left(n_{c} \approx \lambda_{0}^{-2}[\mu \mathrm{m}] \times 1.1 \times 10^{21} \mathrm{~cm}^{-3}\right)$. Since the group velocity increases at lower electron densities, dephasing is often avoided by reducing the plasma density. Yet, this approach has a number of drawbacks. For example, it goes in hand with a reduction of the accelerating field gradient, thus increasing the accelerator length. Furthermore, it is harder to self-guide the laser ${ }^{15}$ since the critical power $P_{c} \sim\left(n_{c} / n_{e}\right) \times 17 \mathrm{GW}^{16}$ It is therefore of interest to find alternatives to mitigate the effects of dephasing. ${ }^{17}$ Such rephasing was recently demonstrated experimentally, ${ }^{18}$ and the aim of this article is to discuss the technique from a theoretical point of view. The paper is structured as follows: First, we discuss energy gain in the self-guided blowout regime. We then discuss the problem of 
phase-locking in this regime and introduce the concept of phase reset. Our analytical estimations are then compared to particle-in-cell (PIC) simulations. We conclude with perspectives for multiple stages of rephasing.

\section{THE SELF-GUIDED HIGHLY NON-LINEAR BLOWOUT REGIME}

Most laser wakefield accelerators rely on self-focusing to guide the laser driver over distances superior to the Rayleigh length. The laser pulse then often focuses and selfcompresses, typically reaching normalized peak field amplitudes in the order of $a_{0} \sim 4-10 .{ }^{19}$ For such intense drivers, the ponderomotive force pushes basically all electrons away, leaving behind a pure ion cavity. ${ }^{20}$ Due to its round shape, this cavity is often referred to as bubble,${ }^{21}$ which is exemplarily shown in Figure 1(a). The cavity scales with the plasma density and the laser intensity. For self-guided laser pulses, it was found empirically that the laser focuses to a matched spot size that follows approximately $k_{p} w_{0} \simeq 2 \sqrt{a_{0}} .{ }^{22}$

For a perfect circular blowout, the potentials inside the bubble have the form

$$
\Phi=\frac{k_{p}^{2}}{4}\left(r_{B}^{2}-r^{2}\right)
$$

and it immediately follows that the associated longitudinal fields are linear

$$
E_{z}=-\frac{m_{e} \omega_{p}^{2}}{2 e}(\pi-\phi) r_{B} .
$$

Here, $\phi$ describes the phase inside the wake. In this definition, the rear part of the bubble corresponds to $\phi=0$, and the center is located at $\phi=\pi$. Note that the above expression is only an approximation. Especially at the rear part of the bubble, the fields take often a non-linear form, depending on the electrons density distribution at this point. We will nonetheless employ Equation (3) in order to estimate the achievable energy gain in the blowout regime. The blowout radius is of the order of the spot size, so for matched conditions $k_{p} r_{B} \simeq 2 \sqrt{a_{0}}$. The maximum accelerating field in this regime is therefore of the order of

$$
\begin{aligned}
E_{z}^{\max } & \sim \frac{m_{e} c \omega_{p}}{e} \sqrt{a_{0}} \\
& \simeq 96 \mathrm{GV} \mathrm{m}^{-1} \times \sqrt{\mathrm{n}_{0}\left[10^{18} \mathrm{~cm}^{-3}\right]} \times \sqrt{a_{0}} .
\end{aligned}
$$

The energy gain of a particle is given by

$$
\Delta \gamma(z)=\frac{q}{m_{e} c_{0}^{2}} \int_{0}^{z} E_{z}\left(z^{\prime}\right) d z^{\prime} .
$$

For a constant phase velocity $v_{\phi}$, assuming that the electron has been injected at the rear $\left(\phi_{0}=0\right)$ and is highly relativistic from this moment on $\left(v_{e} \simeq c_{0}\right)$, we can express the phase shift in the laboratory frame as

$$
\phi(z)=\frac{\pi}{r_{B}}\left(1-\frac{v_{\phi}}{c_{0}}\right) z .
$$

We see from Equation (3) that energy gain will be only achieved until $\phi=\pi$. This is called the dephasing length
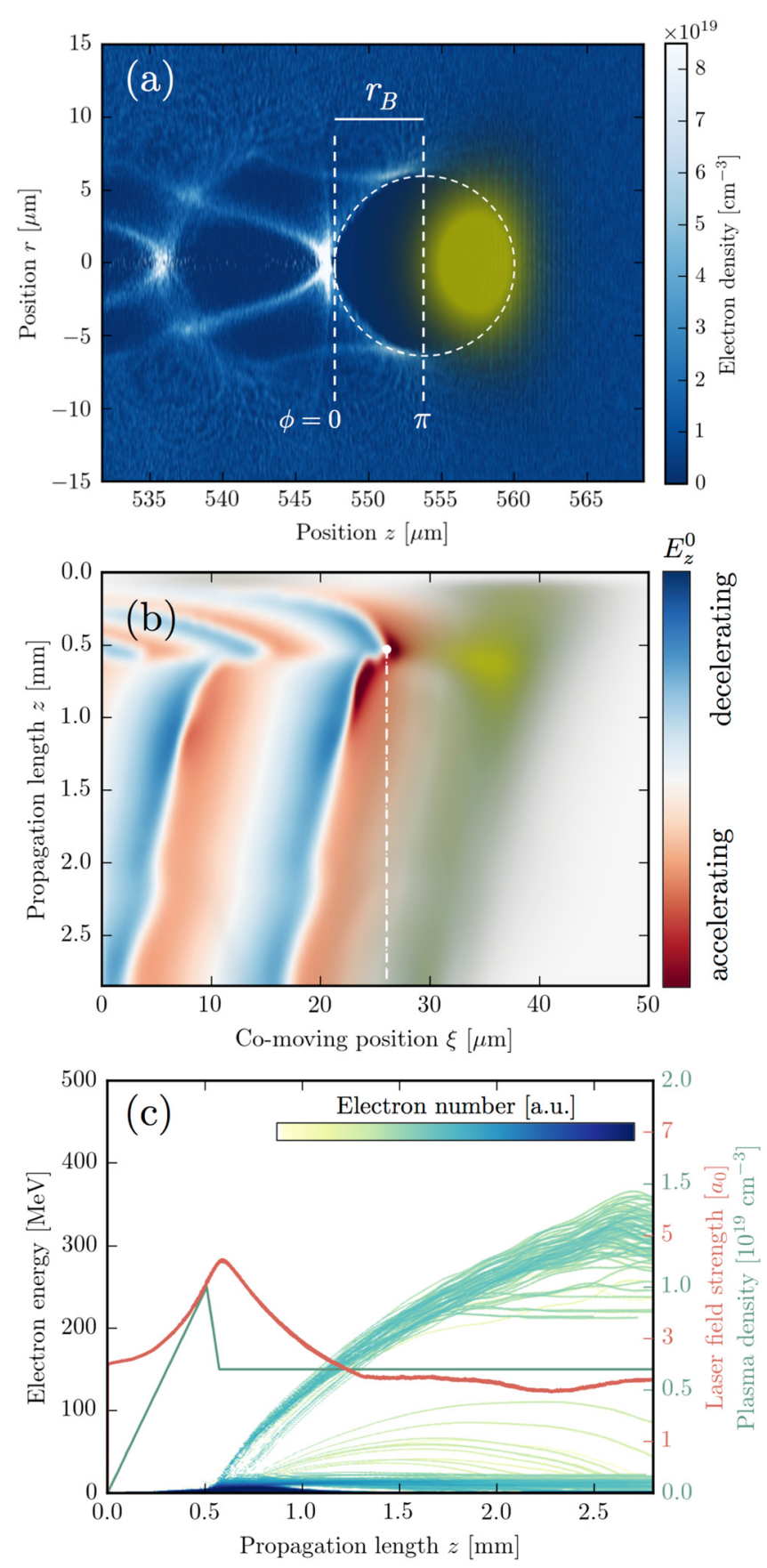

FIG. 1. Simulation of a laser wakefield accelerator in the blowout regime with injection in a density transition. (a) Shows the bubble-shaped ion-cavity (dark blue) that forms behind the laser (yellow). (b) Presents lineouts of the on-axis fields $E_{z}(r=0)$ with accelerating fields in red and decelerating fields in blue. The dashed white line represents the electron bunch, with the dot marking the injection due to the density step, as shown in (c) along with the laser pulse evolution and electron acceleration.

$$
L_{d}=\frac{r_{B}}{1-v_{\phi} / c_{0}} .
$$

Combining these results, we find the energy gain

$$
\begin{aligned}
\Delta \gamma(z) & =\frac{q}{m_{e} c_{0}^{2}} \times E_{z}^{\max } \times \int_{0}^{z}\left(1-z^{\prime} / L_{d}\right) d z^{\prime} \\
& =\Delta \gamma_{\max }\left(2 \frac{z}{L_{d}}-\frac{z^{2}}{L_{d}^{2}}\right),
\end{aligned}
$$


which consists of a rapid linear acceleration in the beginning, which then saturates at $z=L_{d}$, see also Figure 1(c). The maximum gain is $\Delta \gamma_{\max }=\left(q / m_{e} c_{0}^{2}\right) E_{z}^{\max } \times L_{d} / 2$. In general, the gain can be maximized by lowering the plasma density of the accelerator, but as stated in the introduction this has a number of drawbacks and alternatives are desirable.

\section{DENSITY TAPERED LASER-WAKEFIELD ACCELERATORS}

In this section, we discuss the merits of density tapering in order to adapt the phase of the electron beam inside the wakefield. From Equation (6), we see that the phase of electrons in the accelerator is a function of both the phase slippage $\left(1-v_{\phi} / c_{0}\right)$ and the cavity size $r_{B}$. In Sec. II, we have discussed that the scaling laws of the phase velocity suggest to operate at lower plasma densities.

\section{A. Phase-locking}

Alternatively, the phase shift can be compensated by adapting the bubble radius $r_{B}$, ideally maintaining a phase $\phi \sim 0$ to assure the strongest accelerating fields. The cavity size should then change by

$$
\frac{d r_{B}}{d t}=\frac{1}{2} \frac{v_{e}-v_{\phi}}{\left(1-\phi_{0} / 2 \pi\right)} .
$$

The electron velocity can again be assumed $v_{e} \simeq c_{0}$; however, the phase velocity of the plasma wave depends on the laser pulse evolution. In the weakly perturbative nonrelativistic limit $(a \ll 1)$, it is reasonable to use the group velocity $v_{g}$, yet at higher intensities, more effects become relevant, such as pulse steepening and energy depletion. While analytical models extending to the weakly relativistic regime have been proposed, ${ }^{23}$ there exists no model for the blowout regime. It was empirically found that the pulse depletion in this system is of the order of $n_{e} / n_{c}$ lower, ${ }^{22}$ which is equivalent to the etching velocity $v_{\text {etch }}$ in the linear regime. ${ }^{24}$ In general, we can approximate that the phase velocity follows a scaling of the form

$$
\frac{v_{\phi}}{c_{0}} \simeq 1-\kappa \frac{n_{e}}{n_{c}}
$$

with different values of $\kappa$, e.g., $\kappa \simeq 0.5$ for the linear regime or $\kappa \simeq 1.5$ in the blowout regime. The cavity size scales with the plasma wavelength, so starting from a plasma density $n_{e, 0}$ the initial cavity size $r_{B, 0}$ will evolve as $r_{B}=r_{B, 0} \times \sqrt{n_{e, 0} / n_{e}}$. Accordingly,

$$
\frac{d r_{B}}{d t}=\frac{1}{2} \frac{r_{B, 0}}{n_{e, 0}}\left(\frac{n_{e, 0}}{n_{e}}\right)^{3 / 2} \dot{n}_{e}
$$

and Equations (9)-(11) can be combined to a first-order nonlinear ordinary differential equation of the type $\dot{n}_{e}-\alpha \times n_{e}^{5 / 2}=0$, whose solution predicts a scaling

$$
n_{e}(z)=\frac{n_{0}}{\left(1-z / L_{0}\right)^{2 / 3}} .
$$

The density increases first close to linearly, but then the density ramp becomes increasingly steep until a singularity is reached at $L_{0}=(2 / 3 \kappa)(1-\phi / 2 \pi)\left(n_{c} / n_{e, 0}\right) r_{B, 0}$. Using Equations (7) and (10), we find that this length is related to the dephasing length $L_{0}=(2 / 3)(1-\phi / 2 \pi) L_{d}$.

However, in the regime of relativistic optics $\left(a_{0}>1\right)$, the plasma wavelength scales also with the laser intensity, and as mentioned before, the cavity size depends also on the pulse length and width. Yet, the above type of model neglects the laser pulse evolution and the coupling efficiency between the laser pulse and the plasma. For the former, Sprangle and coworkers ${ }^{25}$ have taken into account selffocusing, while Rittershofer et al. ${ }^{26}$ considered the pulse evolution in plasma channels. The coupling efficiency was discussed by Pukhov and Kostyukov. ${ }^{27}$ Unfortunately, all of these approaches are restricted to the linear wakefield regime $\left(a_{0}<1\right)$, and therefore, none of these descriptions are valid for the self-guided bubble regime. Here, it is imperative to include ponderomotive self-focusing, relativistic self-focusing, and self-compression. Increasing the plasma density amplifies these effects. In consequence, the density induced change of the cavity size will be partially or completely counteracted by the augmented laser intensity. Also, the pulse depletion rapidly increases, and increasing energy gain via phase-locking is therefore not straightforward if not possible in this regime. The laser does though not react instantly to the density change, so a sharp transition as in a step-like profile might be a promising alternative.

\section{B. Phase-reset}

Instead of keeping a constant phase $\phi_{0}$, another conceivable situation is that an electron is first accelerated in a flat density profile and the bubble is then forced to diminish at once, so that the particle is again in a region of accelerating fields. For a particle that has dephased to a value $\phi$, the bubble radius has to be reduced to a value $r_{B, 1}=(1-\phi / 2 \pi) r_{B, 0}$ in order to reset the phase to zero. The advantage of this scenario is that if the density transition is sharp enough, we can neglect the laser pulse evolution and assume that the cavity size is determined solely by the plasma density profile. As mentioned before, the scaling is then $r_{B} \propto n_{e}^{-1 / 2}$, so we find that the density $n_{1}$ necessary to achieve the bubble contraction for a phase reset is

$$
n_{1}(\phi)=\frac{n_{0}}{(1-\phi / 2 \pi)^{2}} .
$$

We can now calculate the energy gain for such a phase reset in the blowout regime. For a first estimation, we assume a boost when the electron is just dephased, i.e., $\phi=\pi$ or equivalently $z=L_{d}$. In this case, the required density transition (13) is $n_{1}=4 n_{0}$. Once rephased, the electrons will essentially behave as if they were just injected into a new accelerator with density $n_{1}=4 n_{0}$. So, we can use (8) and sum the dephasing limited gain of those two "stages," which gives

$$
\Delta \gamma_{\max }=\Delta \gamma_{\max }\left(n_{0}\right)+\Delta \gamma_{\max }\left(4 n_{0}\right)=\frac{5}{4} \Delta \gamma_{\max }\left(n_{0}\right) .
$$


The second stage contributes much less to the overall gain, as the dephasing length is shorter at higher density. Still, we expect a gain of around $25 \%$.

Let us now evaluate the optimal case. To find the maximum achievable energy gain in a unique boost, we take the gain in a sawtooth-shaped wakefield (Eq. (8)) up to a position $z_{\text {boost }}$

$$
\Delta \gamma_{1}=\left(\frac{2 z_{\text {boost }}}{L_{d}}-\frac{z_{\text {boost }}^{2}}{L_{d}^{2}}\right) \times \Delta \gamma_{\max }\left(n_{0}\right) .
$$

At $z_{\text {boost }}$, the electron has a phase $\phi_{\text {boost }}=\pi z_{\text {boost }} / L_{d}$, which we can use with (13) to estimate the density necessary to reset the phase at this position. The achievable energy scales inversely with the plasma density, so the gain in this stage will be

$$
\Delta \gamma_{2}=\left(1-\frac{z_{\text {boost }}}{2 L_{d}}\right)^{2} \times \Delta \gamma_{\max }\left(n_{0}\right)
$$

and the complete energy gain is therefore

$$
\Delta \gamma=\left(1+\frac{z_{\text {boost }}}{L_{d}}-\frac{3}{4} \frac{z_{\text {boost }}^{2}}{L_{d}^{2}}\right) \times \Delta \gamma_{\max }\left(n_{0}\right) .
$$

As shown in Figure 2, the global maximum is located before the actual dephasing at $z=2 / 3 \times L_{d}$. It leads to $\Delta \gamma_{\max }\left(\frac{2}{3} L_{d}\right)$ $=\frac{4}{3} \Delta \gamma_{\max }\left(n_{0}\right)$. So, we estimate that a phase reset can lead to

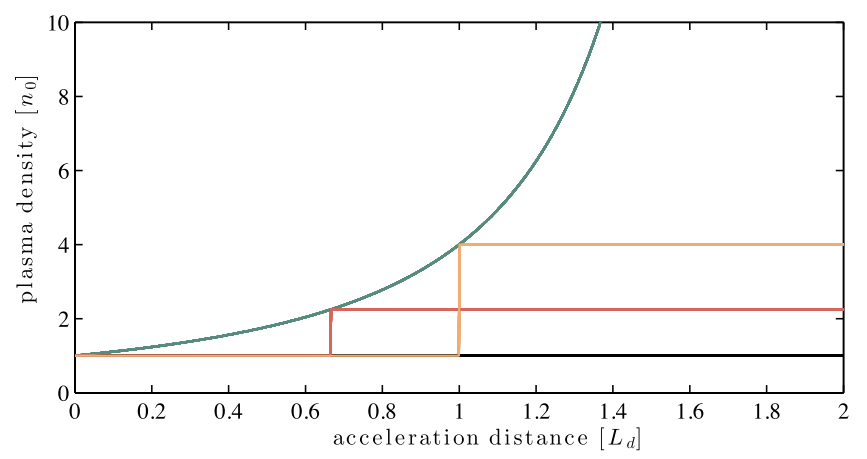

(a)

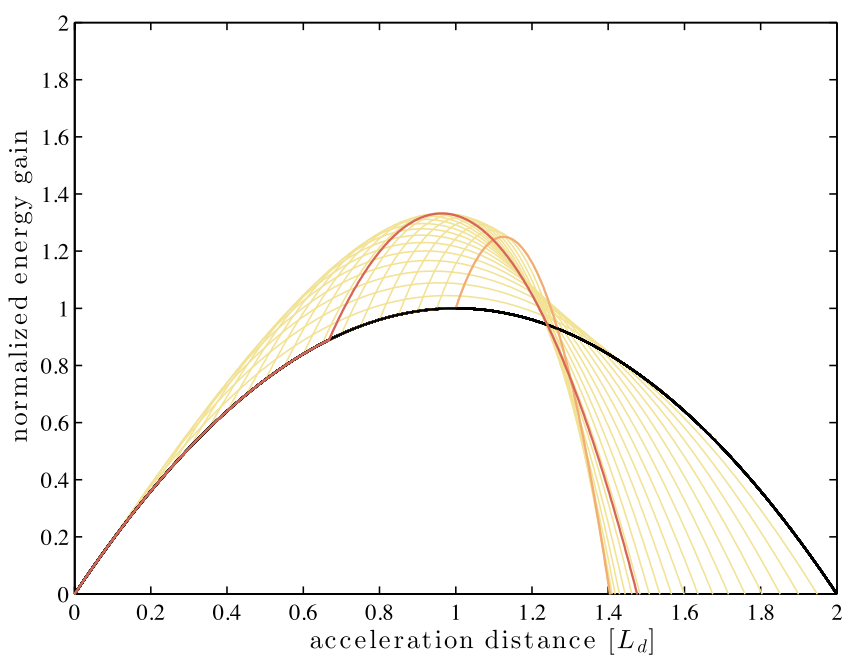

(b)

FIG. 2. Density profiles and estimated energy gain curve for phase-rest to $\phi=0$ at different positions along the acceleration. The density profiles for rephasing at $z=\frac{2}{3} L_{d}$ and $z=L_{d}$ are plotted again in red and orange, respectively. gain in the order of one third of the dephasing limited energy gain. The results are in accordance with Ref. 28, where such a density step scenario was empirically studied using test particle simulations. It is worth noting that this relative energy augmentation is independent of the plasma density $n_{0}$. However, this situation will change if we take into account the laser pulse evolution. As we will discuss in Sec. III C, the scheme is most likely to work best at high densities, where electrons gain significant energy over short distances. Furthermore, nonlinearities in the wakefield, which occur especially at the back of the bubble, may also increase the actual gain of the scheme.

\section{Particle-in-cell simulations}

In order to validate the predictions from the phenomenological model presented in Sec. III B, we have performed three-dimensional particle-in-cell simulations using the quasi-cylindrical code Calder-Circ. ${ }^{29}$ We use two azimuthal modes $(\mathrm{m}=0-1)$ and a $1500 \times 250$ mesh in the longitudinal and radial directions, respectively. The numerical resolution is $\Delta z=0.3 k_{0}^{-1}$, $\Delta r=1.5 k_{0}^{-1}$, and $c_{0} \Delta t=0.96 \Delta z$ (with $k_{0}=1 / \lambda_{0}$ ). The simulation box moves forward at the speed of light in vacuum, so the coordinates $\xi=z-c_{0} t$ are almost copropagating with the laser. Yet, the laser pulse is still slowly dephasing with both electrons (moving also at a velocity close to $c_{0}$ ) and the simulation box. This can be seen, for instance, in Figure 1(b), where it is apparent that the laser pulse (yellow) moves backwards in the simulation box.

The laser pulse is modeled similar to the parameters of the Salle Jaune laser at Laboratoire d'Optique Appliquée, with a duration of 30 femtoseconds, a waist of $11.5 \mu \mathrm{m}$, and a peak intensity $a_{0}=2.5$. In order to avoid beamloading effects, which also alter the cavity size, it is preferential to operate with a weakly charged electron beam. Our reference simulation uses a $50 \mu \mathrm{m}$ density transition from $n_{e}=1 \times 10^{19} \mathrm{~cm}^{-3}$ to $0.6 \times 10^{19} \mathrm{~cm}^{-3}$ to inject a welllocalized electron beam $\left(\sigma_{z}<1 \mu \mathrm{m}\right)$ into the laser wakefield. ${ }^{11}$ Following injection, the density of the reference case remains constant. This density of the plasma is chosen relatively low $\left(n_{e}<10^{19} \mathrm{~cm}^{-3}\right)$ in order to avoid selfinjection, and there is an initial density upramp which prevents injection before the transition. The results of this simulation are shown in Figure 1. We observe that the electrons reach a maximum energy of about $300 \mathrm{MeV}$ after $2 \mathrm{~mm}$ of acceleration, corresponding to an average field gradient of $\sim 150 \mathrm{GV} / \mathrm{m}$.

For the rephasing case, we boost the beam after $z \simeq 0.6 L_{d}$, which according to Equation (13) requires a density increase from $n_{0}$ to $2 n_{0}$. The transition length is $50 \mu \mathrm{m}$. As shown in Figure 3, the density step steepens the energy gain curve, resulting in a final beam energy of around $400 \mathrm{MeV}$. This 30\% increase is very similar to the predictions from the model, see Figure 2. However, as we have discussed in Secs. II and III, after some hundred microns of propagation selffocusing sets in, which then leads to self-injection. The large amounts of electrons injected through this mechanism 

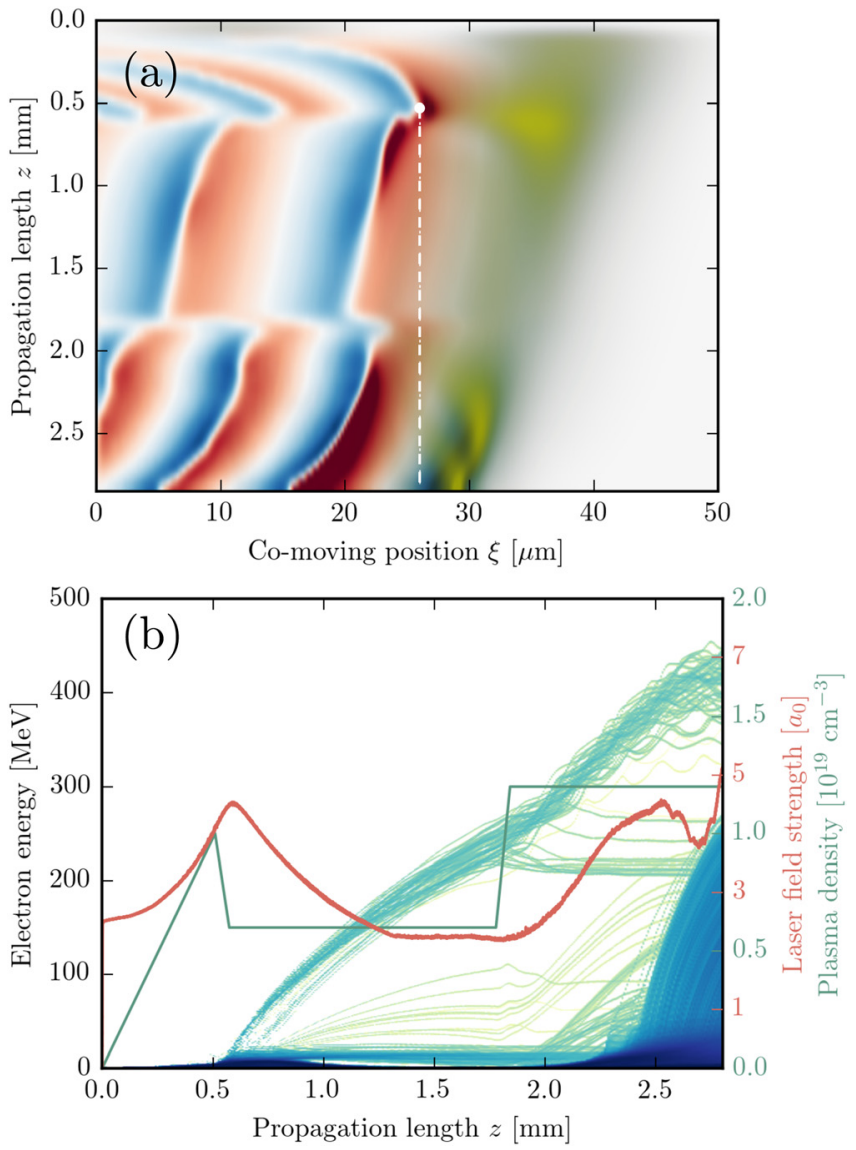

FIG. 3. PIC simulation of electron acceleration with rephasing in a density step. The total gain is about $30 \%$ higher than for the untapered profile.

provoke a cavity expansion which brings the shock injected beam faster into dephasing. We also observe that a small part of the beam is lost during rephasing. This is surprising, as we see in Figure 3(a) that the electron beam (white dashed line) does not reach the rear of the bubble during the rephasing. However, maintaining electron at the rear part of the bubble is delicate because the focusing fields are weak in this region.

In general, we observe that the self-focused laser field strength $a_{0}$ evolves delayed but almost linear with the density profile. In consequence, all cavity contractions in this regime are eventually compensated, and if the density is high enough, self-injection is triggered. We have therefore tested another profile, which reduces the density again after the step. As shown in Fig. 4, the laser pulse evolves much weaker and self-injection is suppressed. The energy gain is reduced in this configuration, yet the gain is still $20 \%$ in this simulation and can be further optimized.

\section{Multiple rephasing stages}

The question arises how much energy gain is achievable in a phase-reset scheme. In Secs. III B and III C, we have seen that the density profile should be tailored in a way that reduces the laser pulse evolution, ${ }^{30}$ which is essentially relativistic self-focusing, ponderomotive self-focusing, and selfcompression.
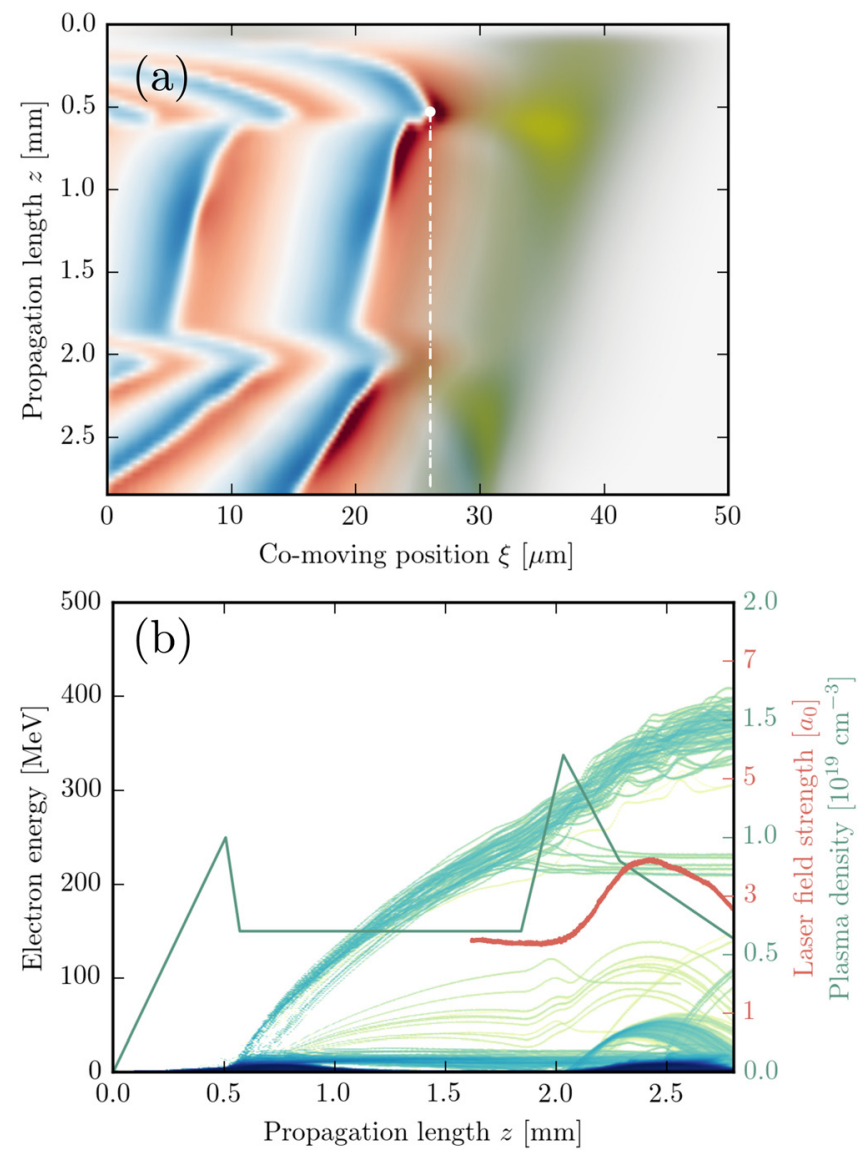

FIG. 4. PIC simulation of electron acceleration with rephasing in a shocklike density profile. The energy gain has reduced to about $20 \%$, but in turn self-injection is suppressed.

As an example, we have calculated the gain that would be achievable in a set of consecutive rephasing stages, each having a sawtooth-like density profile. As shown in Figure 5, it might be possible to achieve more than a twofold increase in the final beam energy in such a configuration. However, for these calculations, we assumed that the laser pulse does not evolve significantly in this profile, which has to be confirmed in particle-incell simulations.

It is also important to point out the differences of rephasing to other concepts of staging in laser-wakefield accelerators. $^{31}$ To date, only single-pulse staging schemes have been experimentally realized, $, 8,32,33$ and none of these schemes does influence the phase between electrons and laser. So, the maximum distance between driver and witness is fixed during injection, which is why the energy gain in such accelerators is basically limited by the injector stage. Increased energy gain would be achievable in multi-pulse setups, where the phase can be reset by use of a new laser pulse that can be synchronized independently of the electron bunch. However, such a setup is much more difficult to realize experimentally since it requires very good alignment and synchronization of the laser and particle beam. Also, additional beam optics such as plasma lenses ${ }^{34,35}$ are required to maintain the electron beam emittance during transport from one stage to the next one. 


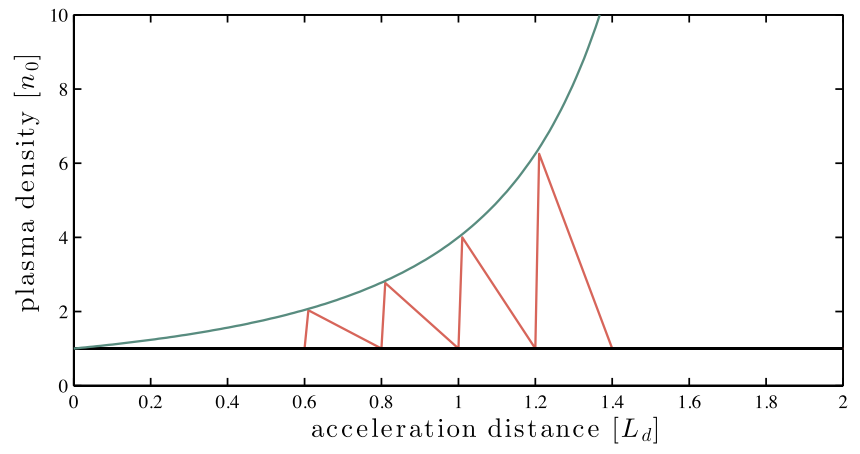

(a)

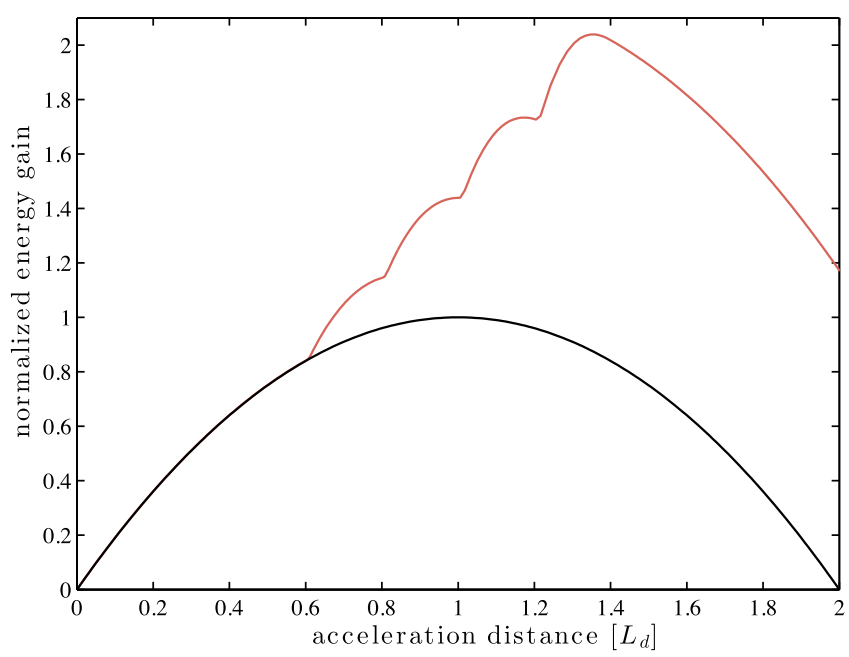

(b)

FIG. 5. Estimation for energy gain via successive rephasing in sawtoothshaped density transitions.

\section{CONCLUSIONS}

In conclusion, we have studied the possibility of increased electron energy gain using density tapering. In contrast to preceding studies, we focused on the phase reset in sharp density transitions. Assuming linear fields inside the blowout region, we estimate that a unique phase reset can lead to a gain in the order of $30 \%$. Similar values are reproduced in particle-in-cell simulations. As seen in Ref. 18, even higher gains are achievable when the fields become non-linear. Furthermore, we have discussed the gain in sawtooth-shaped density profiles which suppresses the laser pulse response to the high plasma densities. We estimate that several stages of such rephasing can lead to a twofold increase in beam energy, which has to be confirmed by comprehensive simulations.

\section{ACKNOWLEDGMENTS}

We acknowledge the Agence Nationale pour la Recherche through the FEN-ICS Project No. ANR-12-JS040004-01, the Agence Nationale pour la Recherche through the FEMTOMAT Project No. ANR-13-BS04-0002, the X-Five ERC Project (Contract No. 339128), EuCARD2/ANAC2 EC FP7 Project (Contract No. 312453), LA3NET Project (GAITN-2011-289191), and GARC Project 15-03118S.

${ }^{1}$ E. Esarey, P. Sprangle, J. Krall, and A. Ting, IEEE Trans. Plasma Sci. 24, 252 (1996).
${ }^{2}$ I. Blumenfeld, C. E. Clayton, F. J. Decker, M. J. Hogan, C. K. Huang, R. Ischebeck, R. Iverson, C. Joshi, T. Katsouleas, N. Kirby et al., Nature 445, 741 (2007)

${ }^{3}$ S. Corde, E. Adli, J. M. Allen, W. An, C. I. Clarke, C. E. Clayton, J. P. Delahaye, J. Frederico, S. Gessner, S. Z. Green et al., Nature 524, 442 (2015).

${ }^{4}$ A. Modena, Z. Najmudin, A. E. Dangor, C. E. Clayton, K. A. Marsh, C. Joshi, V. Malka, C. B. Darrow, C. Danson, D. Neely et al., Nature 377, 606 (1995).

${ }^{5}$ V. Malka, S. Fritzler, E. Lefebvre, M.-M. Aleonard, F. Burgy, J. P. Chambaret, J.-F. Chemin, K. Krushelnick, G. Malka, and S. Mangles, Science 298, 1596 (2002).

${ }^{6}$ W. P. Leemans, B. Nagler, A. J. Gonsalves, C. Toth, K. Nakamura, C. G. R. Geddes, E. Esarey, C. B. Schroeder, and S. M. Hooker, Nat. Phys. 2, 696 (2006).

${ }^{7}$ X. Wang, R. Zgadzaj, N. Fazel, Z. Li, S. A. Yi, X. Zhang, W. Henderson, Y. Y. Chang, R. Korzekwa, H. E. Tsai et al., Nat. Commun. 4, 1 (2013).

${ }^{8}$ H. T. Kim, K. H. Pae, H. J. Cha, I. J. Kim, T. J. Yu, J. H. Sung, S. K. Lee, T. M. Jeong, and J. Lee, Physical Review Letters 111, 165002 (2013).

${ }^{9}$ W. P. Leemans, A. J. Gonsalves, H. S. Mao, K. Nakamura, C. Benedetti, C. B. Schroeder, C. Toth, J. Daniels, D. E. Mittelberger, S. S. Bulanov et al., Phys. Rev. Lett. 113, 245002 (2014).

${ }^{10}$ S. Bulanov, N. Naumova, F. Pegoraro, and J. Sakai, Phys. Rev. E 58, R5257 (1998).

${ }^{11}$ H. Suk, N. Barov, J. B. Rosenzweig, and E. Esarey, Phys. Rev. Lett. 86, 1011 (2001).

${ }^{12}$ E. Esarey, R. F. Hubbard, W. P. Leemans, A. Ting, and P. Sprangle, Phys. Rev. Lett. 79, 2682 (1997).

${ }^{13}$ K. P. Singh, Phys. Plasmas 13, 043101 (2006).

${ }^{14}$ T. P. Wangler, RF Linear Accelerators (John Wiley \& Sons, 2008).

${ }^{15}$ A. J. Gonsalves, K. Nakamura, J. Daniels, H. S. Mao, C. Benedetti, C. B. Schroeder, C. Toth, J. van Tilborg, D. E. Mittelberger, S. S. Bulanov et al., Phys. Plasmas 22, 056703 (2015).

${ }^{16}$ P. Sprangle, C. M. Tang, and E. Esarey, IEEE Trans. Plasma Sci. 15, 145 (1987).

${ }^{17}$ T. Katsouleas, Phys. Rev. A 33, 2056 (1986).

${ }^{18}$ E. Guillaume, A. Döpp, C. Thaury, K. Ta Phuoc, A. Lifschitz, G. Grittani, J. P. Goddet, A. Tafzi, S. W. Chou, L. Veisz et al., Phys. Rev. Lett. 115, 155002 (2015).

${ }^{19}$ S. Corde, A. Lifschitz, G. Lambert, K. T. Phuoc, X. Davoine, R. Lehe, D. Douillet, A. Rousse, V. Malka, and C. Thaury, Nat. Commun. 4, 1501 (2013).

${ }^{20} \mathrm{P}$. Mora and T. M. Antonsen, Phys. Rev. E 53, R2068 (1996).

${ }^{21}$ A. Pukhov and J. Meyer-ter Vehn, Appl. Phys. B 74, 355 (2002).

${ }^{22}$ W. Lu, M. Tzoufras, C. Joshi, F. Tsung, W. Mori, J. Vieira, R. Fonseca, and L. Silva, Phys. Rev. Spec. Top.-Accel. Beams 10, 061301 (2007).

${ }^{23}$ C. Benedetti, F. Rossi, C. B. Schroeder, E. Esarey, and W. P. Leemans, Phys. Rev. E 92, 023109 (2015).

${ }^{24}$ C. D. Decker, W. B. Mori, K. C. Tzeng, and T. Katsouleas, Phys. Plasmas 3, 2047 (1996).

${ }^{25}$ P. Sprangle, B. Hafizi, J. R. Peñano, R. F. Hubbard, A. Ting, C. I. Moore, D. F. Gordon, A. Zigler, D. Kaganovich, and T. M. Antonsen, Phys. Rev. E 63, 056405 (2001).

${ }^{26}$ W. Rittershofer, C. B. Schroeder, E. Esarey, F. J. Grüner, and W. P. Leemans, Phys. Plasmas 17, 063104 (2010).

${ }^{27}$ A. Pukhov and I. Kostyukov, Phys. Rev. E 77, 025401 (2008).

${ }^{28}$ K. T. Phuoc, E. Esarey, V. Leurent, E. Cormier-Michel, C. G. R. Geddes, C. B. Schroeder, A. Rousse, and W. P. Leemans, Phys. Plasmas 15 , 063102 (2008).

${ }^{29}$ A. Lifschitz, X. Davoine, E. Lefebvre, J. Faure, C. Rechatin, and V. Malka, J. Comput. Phys. 228, 1803 (2009).

${ }^{30}$ G. A. Mourou, T. Tajima, and S. V. Bulanov, Rev. Mod. Phys. 78, 309 (2006).

${ }^{31}$ V. Malka, A. Lifschitz, J. Faure, and Y. Glinec, Phys. Rev. Spec. Top.Accel. Beams 9, 091301 (2006).

${ }^{32}$ B. B. Pollock, C. E. Clayton, J. E. Ralph, F. Albert, A. Davidson, L. Divol, C. Filip, S. H. Glenzer, K. Herpoldt, W. Lu et al., Phys. Rev. Lett. 107, 045001 (2011).

${ }^{33}$ J. S. Liu, C. Q. Xia, W. T. Wang, H. Y. Lu, C. Wang, A. H. Deng, W. T. Li, H. Zhang, X. Y. Liang, Y. X. Leng et al., Phys. Rev. Lett. 107, 035001 (2011).

${ }^{34}$ C. Thaury, E. Guillaume, A. Döpp, R. Lehe, A. Lifschitz, K. T. Phuoc, J. Gautier, J. P. Goddet, A. Tafzi, A. Flacco et al., Nat. Commun. 6, 6860 (2015).

${ }^{35}$ J. van Tilborg, S. Steinke, C. G. R. Geddes, N. H. Matlis, B. H. Shaw, A. J. Gonsalves, J. V. Huijts, K. Nakamura, J. Daniels, C. B. Schroeder et al., Phys. Rev. Lett. 115, 184802 (2015). 